\title{
Comparison of Xpert GBS v. culture for rapid detection of group B streptococcus in pregnant women: Sensitivity, specificity and predictive values
}

\author{
M Said, ${ }^{1,2}$ BSc, MB BCh, DTM\&H, FC Path (Microbiology), MMed (Microbiology); Y Dangor, ${ }^{1}$ MSc; \\ N Mbelle, ${ }^{1,2}$ MSc, MB BCh, DTM\&H, FC Path (Microbiology); A Sihlabela, ${ }^{3}$ MB BCh; M R Lekalakala, ${ }^{4,5}$ MB BCh, MMed (Microbiology); \\ F Ismail, ${ }^{1,2}$ BSc, MB BCh, DTM\&H, MMed (Microbiology) \\ ${ }^{1}$ Department of Medical Microbiology, School of Medicine, Faculty of Health Sciences, University of Pretoria, South Africa \\ ${ }^{2}$ National Health Laboratory Service, Tshwane Academic Division, Pretoria, South Africa \\ ${ }^{3}$ Department of Obstetrics and Gynaecology, School of Medicine, Faculty of Health Sciences, University of Pretoria, South Africa \\ ${ }^{4}$ Department of Medical Microbiology, Faculty of Health Sciences, University of Limpopo, Polokwane, South Africa \\ ${ }^{5}$ National Health Laboratory Service, Polokwane, Limpopo, South Africa
}

Corresponding author: M Said (msaid@mweb.co.za)

Background. Group B streptococcus (GBS) is a leading cause of invasive disease, particularly in newborns. Seventy-five percent of neonates will be colonised by mothers carrying the organism. Confirmation of maternal colonisation with GBS is essential for prompt treatment and prevention of neonatal sepsis. The current gold standard of culture for isolation of GBS has a disadvantage of long turnaround time (24 72 hours). Rapid assays are required to determine maternal carriage of GBS.

Objectives. To determine the usefulness of the Xpert GBS technology v. culture methods to detect GBS carriage in pregnant women. Methods. This was a prospective observational study of 284 pregnant women between 26 and 37 weeks' gestation. Two vaginorectal swabs were collected from each participant. One swab was processed using the gold-standard culture method, while the second swab was processed using the Xpert GBS assay. The performance of the Xpert GBS assay was then compared with that of the culture method. Results. Two swabs were processed from each of 284 pregnant women between 26 and 37 weeks' gestation. Culture detected 70 GBS isolates from a total of 279 specimens (25.1\%), whereas the Xpert GBS detected 66 positive specimens (23.7\%). The Xpert GBS assay had a sensitivity of $87 \%$ and specificity of $98 \%$, with a positive predictive value of $92 \%$ and a negative predictive value of $96 \%$.

Conclusions. The Xpert GBS assay is a rapid and sensitive tool for prenatal detection of GBS. The assay should ideally be available in every labour ward, where women can be screened for GBS on arrival.

S Afr Med J 2018;108(12):1032-1035. DOI:10.7196/SAMJ.2018.v108i12.13079

Streptococcus agalactiae or group B streptococcus (GBS) is a leading cause of invasive disease, particularly in newborns. GBS can also cause infections such as chorioamnionitis, endometritis, puerperal sepsis and urinary tract infection in pregnant women, which may be associated with poor fetal outcome. ${ }^{[1,2]}$ Seventy-five percent of neonates will be colonised by mothers carrying the organism. One to two percent of these babies will develop GBS early-onset disease (GBS-EOD), which manifests within the first 7 days of life. ${ }^{[3]}$ In GBSEOD, neonates typically present with sepsis and pneumonia and less frequently with meningitis. The case fatality rate of GBS-EOD ranges from $11 \%$ to $\sim 50 \% .^{[1,2]}$ The incidence of GBS-EOD varies considerably depending on geographical location, but is thought to be $2 / 1000$ live births in South Africa (SA). ${ }^{[4]}$

The Centers for Disease Control and Prevention (CDC) ${ }^{[5]}$ recommends screening pregnant women at 35 - 37 weeks' gestation by obtaining a single vaginorectal swab or separate vaginal and rectal swabs for culture. The swabs should be inoculated into a selective broth medium such as Lim Broth for 18 - 24 hours followed by subculture onto a blood agar plate which is incubated for 18 24 hours and should be observed for beta-haemolytic GBS colonies. ${ }^{[5]}$ Further tests need to be performed to confirm GBS, such as the Christie-Atkins-Munch-Peterson (CAMP) test, hippurate hydrolysis and/or latex agglutination. Women found to be colonised with GBS prenatally are given intrapartum antibiotic prophylaxis (IAP). This measure has been proven to decrease the incidence of GBS-EOD in neonates. ${ }^{[5]}$ According to the $\mathrm{CDC}$, penicillin remains the agent of choice for IAP. ${ }^{[5]}$ Erythromycin and clindamycin are alternatives for penicillin-allergic women and should be administered only after susceptibility results are available. ${ }^{[5]}$ There are no SA guidelines for IAP.

Various selective media are used to isolate GBS. These include CHROMagar StrepB (CHROMagar, France) ChromID Strepto B agar (bioMérieux, France) and the Granada medium (bioMérieux, France). Rosa-Fraile et al. ${ }^{[6]}$ compared the Granada medium with Todd-Hewitt broth followed by subculture on blood agar medium to detect GBS colonisation in pregnant women. The Granada medium was found to be more sensitive than the selective broth for the detection of GBS in vaginorectal samples ( $96 \%$ v. $82 \%$ ). In addition, the use of Granada medium improved the time to reporting of a GBS-positive result by at least 24 hours and reduced the direct cost of screening. ${ }^{[6]}$ A 2014 Japanese study ${ }^{[7]}$ showed that ChromID Strepto $B$ agar had a higher detection rate than blood agar with enrichment. Furthermore, the medium produced fewer false negatives after an extended incubation period. ${ }^{[7]}$ The use of culture as a gold standard to detect GBS colonisation necessitates a longer incubation period of up to 48 hours depending on the culture medium used. This results in a prolonged turnaround time of results, which may lead to delayed 
treatment of patients. In this case, patients at clinics may be sent home to come back for results and are very often lost to follow-up.

To overcome these challenges, more rapid and sensitive techniques have been developed, including molecular assays such as the Xpert GBS (Cepheid, USA). This is a real-time polymerase chain reaction (PCR) assay that amplifies the $c f b$ gene to detect GBS directly from clinical samples with a turnaround time of 37 - 55 minutes ${ }^{[8]}$ Several studies evaluating the Xpert GBS found the assay to have sensitivities ranging from $85 \%$ to $98 \%$ and specificities of between $96 \%$ and $99 \%$ for the detection of GBS colonisation in pregnant women. ${ }^{[8-10]}$ In a landmark study by El-Helali et al. ${ }^{[8]}$ in France, it was found that identification of GBS-positive women in an intrapartum setting can be accurately achieved by the use of the Xpert GBS assay. In comparison with culture as a reference standard, they showed a very high sensitivity of $98.5 \%$ and specificity of $99.6 \%$ for the Xpert GBS assay. ${ }^{[8]}$

\section{Objectives}

To compare the Xpert GBS with culture using Granada medium for the detection of GBS colonisation in pregnant women.

\section{Methods}

\section{Study design and setting}

This was a prospective, observational study conducted at the antenatal clinic at Kalafong Hospital, a tertiary hospital in Pretoria, SA, between November 2013 and May 2014. The study was not registered as a clinical trial.

\section{Participants}

Two hundred and eighty-four pregnant women aged $>18$ years and between 26 and 37 weeks' gestation were enrolled in a convenience series for the study. Participants were only enrolled in the study after written informed consent had been obtained. Baseline demographic characteristics of patients were obtained from a questionnaire that was completed together with the informed consent form. Participants with missing data were excluded from the study.

Eligibility criteria included all pregnant women aged $>18$ years who were $>26$ weeks pregnant. We excluded women who had received antibiotics during the 2 weeks preceding enrolment, those who were part of a GBS vaccine trial and those who refused consent.

Kalafong Hospital has no screening policy for GBS in pregnancy, but a risk-based approach is used. With this approach, any woman presenting in labour with a history of prolonged rupture of membranes (>18 hours), previous GBS colonisation or fever will be prescribed antibiotics intrapartum.

\section{Specimen collection}

Fig. 1 illustrates the laboratory workflow. Vaginorectal swabs were collected using the Copan Venturi Transystem collection device (Copan Diagnostics, USA), which consists of a pair of rayon swabs in Stuart's transport medium. All swabs were collected after excessive secretions/discharge had been removed. The first swab was inserted into the vagina (posterior fornix) and the second into the rectum ( $\sim 2.5 \mathrm{~cm}$ beyond the anal sphincter). All specimens were collected by the same clinician and were transported to the laboratory in a cooler box with ice packs within 4 hours of collection.

\section{GBS culture}

The Cepheid Collection Device consists of a pair of swabs in one device. On arrival at the laboratory, one swab was placed in the Xpert GBS cartridge and run on the GeneXpert module according to the

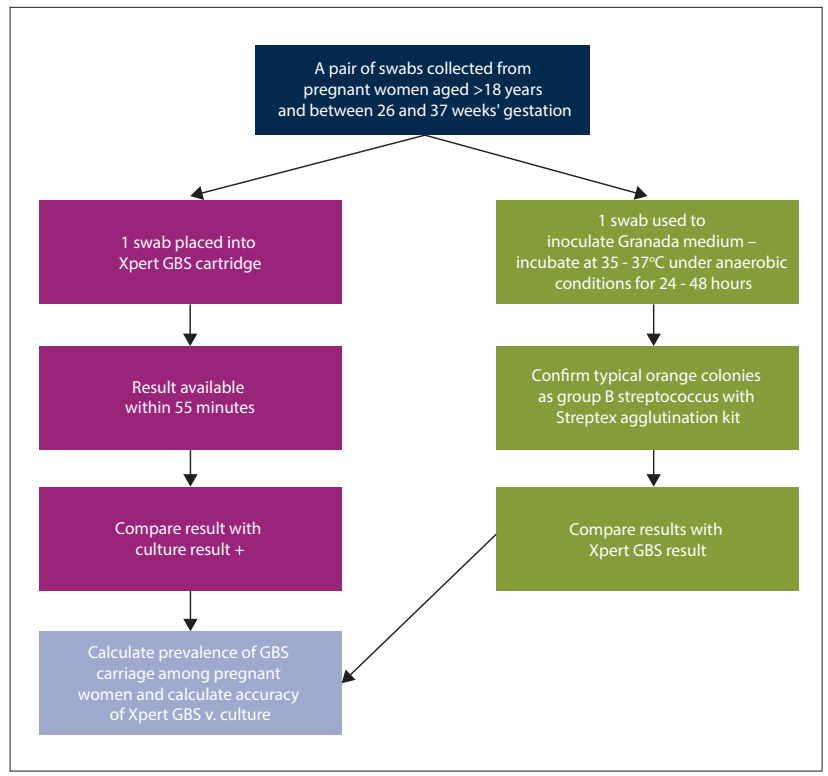

Fig. 1. Diagram illustrating laboratory workflow. $(+=$ positive. $)$

manufacturer's instructions. The second swab was plated on the Granada agar to do a 'head-on' comparison with the Xpert GBS. The Granada medium plates were incubated anaerobically at $35-37^{\circ} \mathrm{C}$ for 24 - 48 hours. Characteristic orange colonies on the Granada medium were confirmed to be GBS by Streptex latex agglutination (Remmel, UK).

\section{Xpert GBS assay}

The second paired vaginorectal swab was placed directly into the Xpert GBS cartridge and processed according to the manufacturer's instructions. If an 'invalid' or 'error' result was generated, the test was repeated using the swab that was used for culture. Results were available between 37 minutes (GBS-positive) and 55 minutes (GBSnegative).

As this was a laboratory-based observational study, positive results on Xpert GBS or culture were not communicated to the clinicians.

\section{Sample size and statistical analysis}

A minimal sample of 246 specimens would estimate the expected prevalence of $20 \%$ GBS colonisation in pregnant women at Kalafong Hospital to an accuracy of within 5\% and a confidence interval of 95\%. The agreement between culture and Xpert GBS was assessed using Cohen's kappa statistic. McNemar's test for symmetry was also determined. Prevalence was reported as a percentage with a 95\% confidence interval. Diagnostic statistics (sensitivity, specificity, positive predictive value (PPV), negative predictive value (NPV)) were also calculated. Indeterminate results from the Xpert GBS were repeated. A subsequent indeterminate result was excluded from the final analysis.

\section{Ethical approval}

Ethical approval for this study was obtained from the Faculty of Health Sciences Research Ethics Committee, University of Pretoria (ref. no. 393/2013).

\section{Results}

\section{Demographic characteristics of participants}

The mean (standard deviation (SD)) age of the study participants was 31.9 (5.57) years, and they had a mean (SD) number of 1.63 (1.05) 
live children; $25.0 \%$ reported a previous stillbirth or miscarriage, while only $5.0 \%$ reported a previous history of preterm labour. The proportion of primigravidas was $13.0 \%$.

\section{Culture v. Xpert GBS}

Two hundred and eighty-four specimens were processed on both the Granada culture medium and Xpert GBS. Forty-three specimens (15.1\%) had to be repeated on the Xpert GBS owing to an 'invalid' result, an 'error' or a 'no result' message from the system. Five samples did not generate a result on Xpert GBS despite repeat testing and were excluded from the analysis. The subsequent 5 unsuccessful attempts included 3 'errors', 1 'invalid' result and 1 'no result'. A total of 279 specimen results were analysed. Interestingly, of the 5 inconclusive samples from Xpert GBS, 3 were negative on culture and 2 were positive.

\section{Prevalence of GBS in pregnancy}

The Granada medium supported the growth of 70 samples (25.1\%), whereas the Xpert GBS detected 66 (23.7\%) (Table 1). Nine samples (3.2\%) were positive by culture but were not detected by Xpert GBS. Xpert GBS detected $5(1.8 \%)$ positives that were not isolated by culture. Group B streptococcus was detected by both methods in 61 samples (21.9\%).

There were 3 instances where orange colonies were observed on the Granada medium but the confirmatory Streptex latex agglutination was negative. Two of the 3 isolates were further identified using the Vitek 2 system and were found to be Leuconostoc mesenteroides ssp. cremoris and $S$. thoraltensis. Both of these isolates were negative on the Xpert GBS. The third isolate was not identified by the Vitek 2 system and was determined to be a bacillus species by 16SrRNA sequencing. This isolate was positive on the Xpert GBS.

\section{Performance of Xpert GBS compared with culture}

Results from the Xpert GBS assay were available as soon as 37 minutes in the case of a positive result, while the maximum time the assay took to give a result was 55 minutes. The time for each individual test was not recorded. The earliest that a culture result was available was 18 hours after incubation. Culture plates were monitored at 18 , 24 and 48 hours for growth; however, the time to positivity for each specimen was not documented.

Using the culture results as a gold standard, the Xpert GBS assay had a sensitivity of $87 \%$ and a specificity of $98 \%$, with a PPV of $92 \%$ and an NPV of $96 \%$. Cohen's kappa statistic was calculated to be 0.8639 , which suggested excellent agreement (95\%). McNemar's test for symmetry was not significant $(p=0.285)$, which suggests that the rates of positivity between the two tests $(25 \%$ v. $24 \%)$ are not statistically significant.

\section{Discussion}

This is the first study that has been undertaken in Africa comparing the Xpert GBS and culture in determining GBS colonisation rates in pregnant women. The colonisation rate with GBS in this study was found to be $25 \%$ by culture and $24 \%$ by Xpert GBS. These findings are comparable with global colonisation rates ranging from $10 \%$ to $40 \% .{ }^{[9]}$ Studies undertaken in SA have shown colonisation rates of $<30 \%$ in Durban ${ }^{[10]}$ and Pretoria (M Chukwu et al., 'Group B streptococcus in pregnant women and their babies at Dr George Mukhari Hospital, Pretoria': unpublished Pathpoint poster presentation, 2012, Cape Town, South Africa). In a more comprehensive study undertaken in Soweto, Johannesburg, GBS colonisation rates were found to be $32.7 \%$ at 26 - 30 weeks' gestation, $28.7 \%$ at $31-35$ weeks and $28.4 \%$ at $\geq 37$ weeks. ${ }^{[11]}$

The Granada medium detected more positive GBS than the Xpert GBS (70 v. 66). The colonies on the medium were easily identified based on the orange colour. In addition, these colonies could be picked off without any difficulty. Twenty-four hours of incubation under anaerobic conditions was adequate and prolonged incubation was not necessary.

Initial Xpert GBS results were not available for 43 specimens (15.1\%). In these cases, the instrument generated messages that included 'error' ( 24 specimens), 'invalid' (12 specimens) and 'no result' (6 specimens). These initial rates where results were unavailable were much higher than the $10.8 \%$ reported by El Helali et al.$^{[8]}$ or the $8.2 \%$ claimed by the manufacturer. However, they were found to be similar to rates reported in a Korean study by Park et al. ${ }^{[12]}$ The major causes of invalid and error results were PCR inhibition and high syringe pressure exceeding the cut-off point, respectively. The presence of significant amounts of mucus or faeces in a sample is known to inhibit PCR and block the microfluidic channel in the cartridge. This effect was minimised in two ways. Firstly, excess mucus/secretions were wiped away at the point of collection, as recommended by the manufacturers. Secondly, a sterile gauze swab was used to wipe away excess mucus/faeces immediately prior to loading the swab into the Xpert GBS cartridge. The high error rate in this study could be a potential disadvantage to routine use of this assay as it would significantly escalate costs when repeat testing is warranted.

The cost of the Xpert GBS cartridge at the time of conducting this study was ZAR300.00 (USD30.45) per cartridge, whereas the cost of culture using Granada agar was ZAR19.00 - 154.00 (USD1.92 - 15.63) per test, depending on whether the CAMP test or latex agglutination was used to confirm the identity of the isolated colonies, respectively. Culture is therefore far cheaper, and this difference in cost will be more pronounced if repeat testing has to be done ( $15 \%$ of samples for Xpert GBS in this study).

The cost of the CDC-recommended culture method for screening for GBS is about the same as using Granada agar. The cost would amount to between ZAR19.75 and ZAR165.00 (USD2.00 - 16.75) per test depending on whether the CAMP test or latex agglutination is used to identify the colonies, respectively. It further has the disadvantage of a longer turnaround time of $48-72$ hours compared with the Granada medium (24 - 48 hours).

The sensitivity of Xpert GBS compared with culture in this study $(87 \%)$ was found to be lower than that detected in three other studies (95.8-98.5\%), ${ }^{[8,13,14]}$ but was comparable to the Korean study, ${ }^{[12]}$ which showed a sensitivity of $86 \%$. The specificity was consistent with other studies. ${ }^{[8,12-14]}$

Nine cases were Xpert GBS-negative and culture-positive. This could be due to the low bacterial load, which would be considered

\section{Table 1. Detection of GBS by culture and Xpert GBS}

Table 1. Detection of GBS by culture and Xpert GBS
\begin{tabular}{llllll} 
Culture- & Xpert GBS- & Xpert GBS-positive, & Xpert GBS-positive, \\
Samples, $\boldsymbol{N}$ & $\begin{array}{l}\text { positive, } \boldsymbol{n}(\%) \\
\text { positive, } \boldsymbol{n}(\%)\end{array}$ & $\begin{array}{l}\text { Xpert GBS-negative, } \\
\text { culture-positive, } \boldsymbol{n}(\%)\end{array}$ & $\begin{array}{l}\text { No results generated } \\
\text { culture-negative, } \boldsymbol{n}(\%)\end{array}$ & $\begin{array}{l}\text { culture-positive, } \boldsymbol{n}(\%) \\
\text { by Xpert GBS, } \boldsymbol{n} \text { (\%) }\end{array}$ \\
\hline $279(25.1)$ & $66(23.7)$ & $61(21.9)$ & $5(1.8)$ & $9(3.2)$ \\
GBS = group B streptococcus. & & & & $5(1.8)$
\end{tabular}


to be below the limit of detection of the assay. The limit of detection reported by Park et al. ${ }^{[12]}$ was 300 colony-forming units per swab. In the 5 cases that were Xpert GBS-positive and culture-negative, it is possible that the Xpert GBS detected the $c f b$ gene in non-viable GBS, or that other organisms such as enterococci hindered the isolation of GBS. Another possible explanation is that the Granada medium does not support the growth of non-haemolytic GBS colonies. Between 1\% and $2 \%$ of GBS colonies are non-haemolytic. ${ }^{[15]}$

Although the Xpert GBS has been evaluated in other countries such as France and Korea, it is not used routinely in clinical practice. ${ }^{[8,12]}$ In SA it is not even currently routine to screen for GBS in pregnancy in the public sector, let alone suggesting a method for screening. In our opinion, the current role of Xpert GBS is therefore for private obstetricians to use the test, either in their rooms or in the labour ward when a woman presents in labour. If GBS screening becomes routine practice in SA, the Xpert GBS could be considered as it can be used on the same machines/modules that are currently used to run the Xpert MTB/Rif test for tuberculosis. However, the cost of the cartridge will determine this.

The major advantage of the Xpert GBS assay is the rapid turnaround time of $37-55$ minutes. It is also relatively easy to perform and therefore possible to use as a potential point-of-care assay. However, in resource-limited settings like SA, culture may be the only option available for the detection of GBS. This study clearly showed that the Granada medium has the advantage of easy and early detection of GBS with no significant escalation in the cost of culture.

\section{Study limitations}

A limitation was that this was a single-centre study. As a result, there could be a potential source of bias in the selection of patients. Another potential source of bias may be that participants were only selected from the antenatal clinic and not from other sites at Kalafong Hospital such as the labour ward or the obstetrics and gynaecology wards. A further limitation was that the exact running time for each Xpert GBS test was not recorded. As a result, we could not determine factors such as the median duration and interquartile range for this test. In addition, participants with missing data could have been traced back to retrieve the data. A shortcoming of the study design was that it was purely a laboratory-based study comparing two assays.

\section{Conclusions}

Compared with the standard culture method, the Xpert GBS assay is a rapid and sensitive tool for prenatal detection of GBS. Ideally, it is a point-of-care test that should be used in the labour ward when a patient presents in labour. In view of the high primary error rate
(17\%) experienced in our study, the financial feasibility of potential uses will need to be evaluated further.

Declaration. The research described in this article was an MMed project of MS, the main author.

Acknowledgements. Prof. Anwar Hoosen, University of the Free State, critically revised the final draft of the manuscript.

Author contributions. MS: collection of specimens, laboratory processing of specimens, analysis of data, writing up of article; YD: laboratory processing of specimens, analysis of data; NM: writing up of article; AS: collection of specimens; MRL: writing up of article; FI: collection of specimens, laboratory processing of specimens, analysis of data, writing up of article.

Funding. The research was funded by a National Health Laboratory Services Research Grant (no. 94443) in fulfilment of MS’s MMed project.

Conflicts of interest. None.

\footnotetext{
1. Winn HJ. Group B streptococcus infection in pregnancy. Clin Perinatol 2007;34(3):387-392. https:// doi.org/10.1016/..clp.2007.03.012

2. Koenig JM, Keenan WJ. Group B streptococcus and early onset sepsis in the era of maternal prophylaxis. Pediatr Clin North Am 2009;56(3):689-708. https://doi.org/10.1016/.jpcl.2009.04.003

3. Chan SHS, Wan KH, Lee WH. Review on group B streptococcus infection. Hong Kong J Paediat 2000;31(5):166-174.

4. Madhi SA, Radebe K, Crewe-Brown H, et al. High burden of invasive Streptococcus agalactiae disease in South African infants. Ann Trop Paediatr 2003,23(1):15-23. https://doi. org/10.1179/000349803125002814

5. Verani JR, McGee L, Schrag SJ. Prevention of perinatal group B streptococcal disease: Revised guidelines from CDC, 2010. MMWR 2010;59(RR-10):1-32. https://www.cdc.gov/mmwr/preview/ mmwrhtml/rr5910al.htm (accessed 19 October 2018).

6. Rosa-Fraile M, Rodriguez-Granger J, Cueto-Lopez M, et al. Use of Granada medium to detect group B streptococcal colonization in pregnant women. J Clin Microbiol 1999;37(8):2674-2677.

7. Morita T, Feng D, Kamio Y, et al. Evaluation of ChromID Strepto B as a screening media for Streptococcus agalactiae. BMC Infect Dis 2014;14:46. https://doi.org/10.1186/1471-2334-14-46

8. El Helali N, Nguyen JC, Ly A, et al. Diagnostic accuracy of a rapid real-time polymerase chain reaction assay for universal intrapartum group B streptococcus screening. Clin Infect Dis 2009;49(3):417-423. https://doi.org/10.1086/600303

9. Picard FJ, Bergeron M. Laboratory detection of group B streptococcus for prevention of perinatal disease. Eur J Clin Microbiol Infect Dis 2004;23(9):665-671. https://doi.org/10.1007/s10096-004-1183-8

10. Hallett AF, Govender P, Pillay SS, et al. Group B streptococci in blacks. S Afr Med J 1979;55(5):157-159.

10. Hallett AF, Govender P, Pillay SS, et al. Group B streptococci in blacks. S Afr Med ) 1979;55(5):157-159.
11. Kwatra G, Adrian PV, Shiri T, et al. Serotype-specific acquisition and loss of group B streptococcus Kwatra G, Adrian PV, Shiri T, et al. Serotype-specific acquisition and loss of group B streptococcus
recto-vaginal colonisation in late pregnancy. Plos One 2014;9(6):e98778. https://doi.org/10.1371/ ournal.pone.0098778

12. Park J, Cho D, Yang J, et al. Usefulness of a rapid real-time PCR assay in prenatal screening for group B streptococcus colonization. Ann Lab Med 2013;33(1):39-44. https://doi.org/10.3343/alm.2013.33.1.39

13. Church DL, Baxter H, Lloyd T, et al. Evaluation of the Xpert group B streptococcus real-time polymerase chain reaction assay compared to StrepB Carrot Broth for the rapid intrapartum detection of group B streptococcus colonization. Diagn Microbiol Infect Dis 2011;69(4):460-462. https://doi. org/10.1016/j.diagmicrobio.2010.11.002

14. Gavino M, Wang E. A comparison of a new rapid real-time polymerase chain reaction system to traditional culture in determining group B streptococcus colonization. Am J Obstet Gynecol 2007;197(4):388.e1-4. https://doi.org/10.1016/.j.ajog.2007.06.016

15. Edwards MS, Baker CJ. Streptococcus agalactiae (group B streptococcus). In: Bennett JE, Dolin R, Blaser MJ, eds. Mandell, Douglas and Bennett's Principles and Practice of Infectious Diseases. 8th ed. Philadelphia: Elsevier Saunders, 2015:2340-2347.
}

Accepted 18 May 2018. 